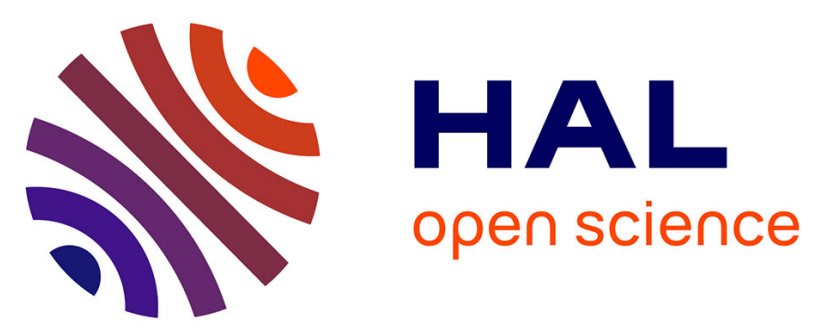

\title{
An integrated control strategy to solve the disturbance decoupling problem for max-plus linear systems with applications to a high throughput screening system
} Ying Shang, Laurent Hardouin, Mehdi Lhommeau, Carlos-Andrei Maia

\section{- To cite this version:}

Ying Shang, Laurent Hardouin, Mehdi Lhommeau, Carlos-Andrei Maia. An integrated control strategy to solve the disturbance decoupling problem for max-plus linear systems with applications to a high throughput screening system. Automatica, 2016, 63, pp.338-348. 10.1016/j.automatica.2015.10.030 . hal-01229626

\section{HAL Id: hal-01229626 \\ https://hal.science/hal-01229626}

Submitted on 26 Sep 2019

HAL is a multi-disciplinary open access archive for the deposit and dissemination of scientific research documents, whether they are published or not. The documents may come from teaching and research institutions in France or abroad, or from public or private research centers.
L'archive ouverte pluridisciplinaire HAL, est destinée au dépôt et à la diffusion de documents scientifiques de niveau recherche, publiés ou non, émanant des établissements d'enseignement et de recherche français ou étrangers, des laboratoires publics ou privés. 


\title{
An Integrated Control Strategy to Solve the Disturbance Decoupling Problem for Max-Plus Linear Systems with Applications to a High Throughput Screening System *
}

\author{
Ying Shang a , Laurent Hardouin ${ }^{\text {b }}$, Mehdi Lhommeau ${ }^{\text {b }}$, Carlos Andrey Maia ${ }^{\text {c }}$

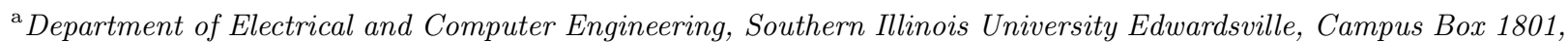 \\ Edwardsville, IL 62026, USA
}

b Laboratoire Angevin de Recherche en Ingénierie des Systèmes of the University of Angers, 62 avenue Notre Dame du Lac, 49000 Angers, France

${ }^{\mathrm{c}}$ Departamento de Engenharia Elétrica, Universidade Federal de Minas Gerais (UFMG). Av. Antônio Carlos 6627, Pampulha, 31270-010, Belo Horizonte, MG, Brazil

\begin{abstract}
This paper presents the new investigations on the disturbance decoupling problem (DDP) for the geometric control of maxplus linear systems. The classical DDP concept in the geometric control theory means that the controlled outputs will not be changed by any disturbances. In practical manufacturing systems, solving for the DDP would require further delays on the output parts than the existing delays caused by the system breakdown. The new proposed modified disturbance decoupling problem (MDDP) in this paper ensures that the controlled output signals will not delayed more than the existing delays caused by the disturbances in order to achieve the just-in-time optimal control. Furthermore, this paper presents the integration of output feedback and open-loop control strategies to solve for the MDDP, as well as for the DDP. If these controls can only solve for the MDDP, but not for the DDP, an evaluation principal is established to compare the distance between two output signals generated by controls solving for the MDDP and DDP, respectively. This distance can be interpreted as the number of tokens or firings that are needed in order for the controls to solve for the DDP. Moreover, another alternative approach is finding a new disturbance mapping in order to guarantee the solvability of the DDP by the same optimal control for the MDDP. The main results of this paper are illustrated by using a timed event graph model of a high throughput screening system in drug discovery.
\end{abstract}

Key words:

(Max,+)-algebra, discrete-event systems, geometrical control theory, manufacturing systems.

\section{Introduction}

Max-plus linear systems $([1,11,15])$ are used to model timed discrete event systems. The main advantage of max-plus linear systems is incorporating the traditional linear system theory in modeling and analysis of the nonlinear synchronization behaviors in discrete event systems. They are suitable to describe algebraically the behaviors of timed event graphs(TEGs). A TEG is a sub-

\footnotetext{
* Corresponding author Ying Shang. Tel. +1-618-650-2800. Fax +1-618-650-3374.

Email addresses: yshang@siue.edu (Ying Shang), laurent.hardouin@univ-angers.fr (Laurent Hardouin), mehdi.lhommeau@univ-angers.fr (Mehdi Lhommeau), maia@cpdee.ufmg.br (Carlos Andrey Maia).
}

class of timed Petri net models for discrete event systems in which each place only has a single upstream transition and a single downstream transition. Over the past three decades, many fundamental problems for max-plus linear systems have been studied by researchers, for example, controllability ([23]), observability ([12]), and the model reference control problem ([20]). However, the geometric theory for max-plus linear systems introduced in ([7]) has not been well established as the traditional linear systems $([2,26])$. Only a few existing research results on generalizing fundamental concepts and problems in geometric control are generalized to max-plus linear systems, such as computation of different controlled invariant sets $([19,14,21])$ and the disturbance decoupling problem $([17])$. 
This paper reports upon recent investigations on the disturbance decoupling problem (DDP) for max-plus linear systems, which means the output signals remain unchanged in the presence of the disturbances. For maxplus linear systems, a disturbance is an event which blocks the occurrence of an event (in manufacturing setting, it could be a machine breakdown or a delay in a component supply) and the control consists in choosing the date of an input event (e.g. when a job should be started on a machine). Hence, solving for the DDP means finding input dates such that outputs will be delayed more than the delays caused by the disturbances. From a practical point of view, it should be more interesting to find a control such that the system is not delayed more than the delays caused by the disturbances. For example, when a system breakdown occurs, we can put the input parts of the manufacturing line as late as possible to reduce the unnecessary waiting time in the network, but not too late in order not to degrade the performance. Therefore, the modified disturbance decoupling problem (MDDP) in $([13,25])$ is to find appropriate controls such that the output signals will not be delayed more than the outputs caused by the disturbances. In ([13]), the solvability conditions for the DDP are presented, as well as the state feedback controls solving for the MDDP. In ([25]), an open-loop control is presented to solve for the MDDP, and such a control can solve for the DDP at the same time if and only if the output images of the reachable space of the disturbances for the open-loop systems are subsets of the output images of the reachable space of the open-loop controls.

Hence, the aim of this paper is to present an integration of the output feedback controls ([13]) and the open-loop controls $([25])$ such that the MDDP can be solved. Furthermore, it will be shown that this pair of controls solves for the DDP if and only if the output image of the reachable space of the disturbances for the open-loop system is a subset of the output image of the reachable space of the open-loop control. When this necessary and sufficient condition is not satisfied, so the integrated strategy can only solve the MDDP, but not the DDP, then an evaluation principle is established to compare the distance, which is interpreted as the event delays between the output signals generated by the controllers and the output signals generated by the disturbances. Alternatively, if the disturbance is measurable ${ }^{1}$, a new control strategy is developed in order to guarantee the solvability of the DDP by using the same control as for the MDDP. This original control strategy yields a manner to modify how the disturbances act on the system such that the disturbance can be rejected. If you consider disturbances as component supply disruptions, the strategy gives the minimal number of rough parts you need on the shelve to be able to solve the DDP.

\footnotetext{
1 This assumption is practically reasonable in manufacturing setting, because the component supply disruptions are generally known, and can be measured quite easily.
}

The remainder of this paper is organized as follows. Section 2 presents the mathematical preliminaries in maxplus algebra literature. Section 3 defines the max-plus linear system models and introduces the concepts of the DDP and the MDDP in max-plus linear systems. Section 4 presents the event domain approach to find the integrated controls solving for the MDDP and the DDP, respectively. If the integration of the state-feedback control and the open-loop control can only solve for the MDDP, not for the DDP, a distance evaluation is presented in Section 5 between the two output trajectories solving for the DDP and the MDDP, and a new disturbance mapping is established in Section 6 such that the integrated controls will solve for the DDP and the MDDP, simultaneously. The main results of this paper are illustrated by a high throughput screening system in drug discovery in Section 7. Section 8 concludes this paper with future research directions.

\section{Mathematical Preliminaries}

A semiring is a set $\mathcal{S}$, equipped with two operations $\oplus, \otimes$, such that $(\mathcal{S}, \oplus)$ is a commutative monoid (the zero element will be denoted $\varepsilon),(\mathcal{S}, \otimes)$ is a monoid (the unit element will be denoted $e$ ), operation $\otimes$ is right and left distributive over $\oplus$, and $\varepsilon$ is absorbing for the product (i.e. $\varepsilon \otimes a=a \otimes \varepsilon=\varepsilon, \forall a$ ). A semiring $\mathcal{S}$ is idempotent if $a \oplus a=a$ for all $a \in \mathcal{S}$. A non empty subset $\mathcal{B}$ of a semiring $\mathcal{S}$ is a subsemiring of $\mathcal{S}$ if for all $a, b \in \mathcal{B}$ we have $a \oplus b \in \mathcal{B}$ and $a \otimes b \in \mathcal{B}^{2}$. In this paper, we denote $\overline{\mathbb{Z}}_{\max }=(\mathbb{Z} \cup\{-\infty,+\infty\}, \max ,+)$ as the integer maxplus semiring.

In an idempotent semiring $\mathcal{S}$, operation $\oplus$ induces a partial order relation

$$
a \succeq b \Longleftrightarrow a=a \oplus b, \quad \forall a, b \in \mathcal{S} .
$$

Then, $a \vee b=a \oplus b$. An idempotent semiring $\mathcal{S}$ is complete if sums of infinite numbers of terms are always defined, and if multiplication distributes over infinite sums too. In particular, the sum of all the elements of the idempotent semiring is denoted $\top$ (for 'top'). A mapping $f: \mathcal{S} \rightarrow \mathcal{S}$, where $\mathcal{S}$ is a complete idempotent semiring, is residuated if and only if $f(\varepsilon)=\varepsilon$ and $f$ is lower-semicontinuous, that is,

$$
f\left(\bigoplus_{i \in I} a_{i}\right)=\bigoplus_{i \in I} f\left(a_{i}\right)
$$

for any (finite or infinite) set $I$. The mapping $f$ is said to be residuated and $f^{\sharp}$ is called its residual. It is straightforward that $L_{a}: \mathcal{S} \rightarrow \mathcal{S}, x \mapsto a x$ and $R_{a}: \mathcal{S} \rightarrow \mathcal{S}, x \mapsto$ $x a$ are lower semi-continuous. Therefore these mappings

\footnotetext{
${ }^{2}$ As in the conventional algebra, the multiplication $\otimes$ is often omitted.
} 
are both residuated i.e., $L_{a}(x) \preceq b\left(\operatorname{resp} . R_{a}(x) \preceq b\right)$ admits a greatest solution $\top$, then the following notations are considered :

$$
\begin{aligned}
& L_{a}^{\sharp}(b)=a \phi b=\bigoplus\{x \mid a x \preceq b\} \text { and } \\
& R_{a}^{\sharp}(b)=b \phi a=\bigoplus\{x \mid x a \preceq b\}, \quad \forall a, b \in \mathcal{S},
\end{aligned}
$$

where $L_{a}^{\sharp}$ is called the residual mappings, and is the unique mapping such that $L_{a} \circ L_{a}^{\sharp} \preceq I d$ and $L_{a}^{\sharp} \circ L_{a} \succeq I d$ where $I d$ is the identity mapping (the same holds for $\left.R_{a}\right)$. The implicit equation $x=a x \oplus b$ admits $x=a^{*} b=$ $\left(\bigoplus_{k>0} a^{k}\right) b$ as smallest solution. All these results admit a natural extension to the matrix case, where the sum and product of matrices are defined with the same rules as in classical theory (see [1]).

Definition $1([\mathbf{5}, \mathbf{6}, \mathbf{8}])$ Let $\mathcal{S}$ be a complete idempotent semiring and let $C$ be $a \times p$ matrix with entries in $\mathcal{S}$. We call null kernel of $C$ as the set of elements $x \in \mathcal{S}^{p}$ such that $C x=\varepsilon$, denoted as $\operatorname{ker} C$. We call equivalence kernel of $L_{C}$ (denoted by $\operatorname{ker}_{\mathrm{eq}} C$ ), the subset of all pairs of elements of $\mathcal{S}^{p}$ whose components are both mapped by $L_{C}$ to the same element in $\mathcal{S}^{n}$, i.e., the following definition

$$
\operatorname{ker}_{\text {eq }} C:=\left\{\left(s, s^{\prime}\right) \in\left(\mathcal{S}^{p}\right)^{2} \mid C s=C s^{\prime}\right\} \text {. }
$$

Clearly $\operatorname{ker}_{\mathrm{eq}} C$, is an equivalence relation on $\mathcal{S}^{p}$, i.e., $C s=C s^{\prime} \Longleftrightarrow s^{\prime} \equiv s\left(\bmod \operatorname{ker}_{\mathrm{eq}} C\right)$ and furthermore it is a congruence and then we can define the quotient $\mathcal{S}^{p} / \operatorname{ker}_{e q} C$.

The subset of elements $s^{\prime} \in \mathcal{S}^{p}$ that are equivalent to $s$ modulo $\operatorname{ker}_{e q} C$ is denoted as $[s]_{C}$, i.e.,

$$
[s]_{C}=\left\{s^{\prime} \in \mathcal{S}^{p} \mid s^{\prime} \equiv s\left(\bmod \operatorname{ker}_{\mathrm{eq}} C\right)\right\} \subset \mathcal{S}^{p} .
$$

Definition 2 (Restricted map) Let $f: \mathcal{S}^{p} \rightarrow \mathcal{S}^{n}$ be a map and $\mathcal{A} \subseteq \mathcal{S}^{p}$. We will denote ${ }^{3} f_{\mid \mathcal{A}}: \mathcal{A} \rightarrow \mathcal{S}^{n}$ the map defined by $f_{\mid \mathcal{A}}=f \circ$ old $_{\mid \mathcal{A}}$ where $\operatorname{ld}_{\mid \mathcal{A}}: \mathcal{A} \rightarrow \mathcal{S}^{p}, x \mapsto x$ be the canonical injection. Identically, let $\mathcal{B} \subseteq \mathcal{S}^{n}$ with $\operatorname{Im} f \subseteq \mathcal{B}$. Map $_{\mathcal{B} \mid} f: \mathcal{S}^{p} \rightarrow \mathcal{B}$ is defined by $f=\overline{\operatorname{Id}}_{\mid \mathcal{B}} \circ{ }_{\mathcal{B} \mid} f$, where $\operatorname{ld}_{\mid \mathcal{B}}: \mathcal{B} \rightarrow \mathcal{S}^{n}, x \mapsto x$ be the canonical injection.

Definition 3 (Isotone map) A map $f: \mathcal{S}^{p} \rightarrow \mathcal{S}^{p}$ is said to be order preserving or isotone if the following property holds: $a \preceq b \Rightarrow f(a) \preceq f(b)$.

Definition 4 (Closure map) An isotone map $f$ : $\mathcal{S}^{p} \rightarrow \mathcal{S}^{p}$ is a closure map if $f \succeq \mathrm{Id}_{\mathcal{S}^{p}}$ and $f \circ f=f$.

Proposition 1 ([10]) A closure map $f: \mathcal{S}^{p} \rightarrow \mathcal{S}^{p}$ restricted to its image $e_{\operatorname{Im} f \mid} f$ is a residuated map whose residual is the canonical injection $\operatorname{Id} \mid \operatorname{Im} f: \operatorname{Im} f \rightarrow \mathcal{S}^{p}, s \mapsto s$.

\footnotetext{
3 These notations are borrowed from classical linear system theory see [26].
}

Corollary 1 Let $\mathcal{K}: \mathcal{S}^{p} \rightarrow \mathcal{S}^{p}, s \mapsto s^{*}$ be a map, where $s^{*}=\bigoplus_{i \in \mathbb{N}} s^{i}$ (see Appendix A for complementary results on map $\mathcal{K})$. The $\operatorname{map}_{\operatorname{Im} \mathcal{K} \mid} \mathcal{K}$ is a residuated map whose

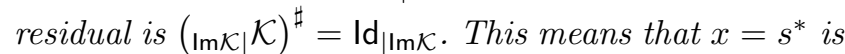
the greatest solution to inequality $x^{*} \preceq s^{*}$. Actually, the greatest solution achieves equality.

\section{Disturbance Decoupling Problem (DDP) of Max-Plus Linear Systems}

A max-plus linear system is defined by the following equations

$x(k)=A x(k-1) \oplus B u(k) \oplus S q(k)$,

$y(k)=C x(k)$,

where $x(k) \in \overline{\mathbb{Z}}_{\text {max }}^{n \times l}, u(k) \in \overline{\mathbb{Z}}_{\max }^{p \times l}, q(k) \in \overline{\mathbb{Z}}_{\max }^{r \times l}, y(k) \in$ $\overline{\mathbb{Z}}_{\max }^{q \times l}$ and $k \in \mathbb{Z}$. This kind of system makes it possible to describe the behaviors of TEGs, by associating to each transition a firing date sequence $x_{i}(k) \in \overline{\mathbb{Z}}_{\max }^{1 \times l}$ and predict the system evolution thanks to Eq. (3).

\subsection{Disturbance Decoupling Problem (DDP)}

Definition 5 The system (3) is called disturbance decoupled by an output feedback control $u(k)=F y(k) \oplus$ $v(k)$ (or by an open-loop control $u(k)=v(k)$ ) if and only if any disturbance signal will not affect the system output $y(k)$ for all $k \in \mathbb{Z}$ and for any initial condition $x_{0}$.

Proposition 2 ([13]) Given a max-plus linear system of the form (3), the DDP is solvable by an open-loop controlu $(k)$ if and only if there exists an open-loop control sequence $\vec{u}=[u(m) u(m-1) \cdots u(1)]^{T}$ such that the equivalence relation holds

$\langle A \mid B\rangle_{m} \vec{u} \oplus\langle A \mid S\rangle_{m} \vec{q} \equiv\langle A \mid B\rangle_{m} \vec{u}\left(\bmod \operatorname{ker}_{\mathrm{eq}} C\right)$

where

$$
\langle A \mid S\rangle_{m}=\left[\begin{array}{llll}
S A S & \cdots & A^{(m-1)} S
\end{array}\right]
$$

and

$$
\langle A \mid B\rangle_{m}=\left[\begin{array}{llll}
B & A B & \cdots & A^{(m-1)} B
\end{array}\right]
$$

for all $m$ and any disturbance signal

$$
\vec{q}=[q(m) q(m-1) \cdots q(1)]^{T}
$$

Proposition 3 ([13]) Given a max-plus linear system of the form (3), the DDP is solvable by an output feedback control $u(k)=F y(k) \oplus v(k)$ for $v(k) \neq \epsilon$ if and only if there exist an output feedback mapping $F$ and a 
control sequence $\vec{v}=[v(m) v(m-1) \cdots v(1)]^{T}$ such that the equivalence relation holds

$$
\begin{aligned}
& \langle A \oplus B F C \mid B\rangle_{m} \vec{v} \oplus\langle A \oplus B F C \mid S\rangle_{m} \vec{q} \\
& \equiv\langle A \oplus B F C \mid B\rangle_{m} \vec{v}\left(\bmod \operatorname{ker}_{\mathrm{eq}} C\right)
\end{aligned}
$$

for all $m$ and any disturbance signal $\vec{q}=\left[\begin{array}{lll}q(m) & \cdots & q(1)\end{array}\right]^{T}$, where $\langle A \oplus B F C \mid S\rangle_{m}=S \oplus(A \oplus B F C) S \oplus \cdots \oplus$ $(A \oplus B F C)^{(m-1)} S$ and $\langle A \oplus B F C \mid B\rangle_{m}=B \oplus(A \oplus$ $B F C) B \oplus \cdots \oplus(A \oplus B F C)^{(m-1)} B$.

Propositions 2 and 3 indicate that, in order to check the solvability condition for the DDP of max-plus linear systems, we need an infinite number of checking for the equations (4) and (5), respectively. Moreover, solving for the DDP of max-plus linear systems implies that we have to delay the process at least the same as the disturbance has placed on the system. Hence the disturbance has no more influences on the output, it is like the disturbance was hidden by the control input. Therefore, the traditional DDP definition is very restrictive for max-plus linear systems, a modified DDP with better practical meanings will be introduced in the following subsection.

\subsection{Modified Disturbance Decoupling (MDDP)}

From the practical point of view, a modified DDP for max-plus linear systems is defined as follows:

Definition 6 The max-plus linear system described in Eq. (3) is called modified disturbance decoupled by an output feedback control $u(k)=F y(k) \oplus v(k)$ (or an open-loop control $u(k)=v(k))$ if and only if the system output signals will not be disturbed more than the output signals influenced by the disturbances.

Moreover, MDDP means that the control will delay the token inputs as much as possible in order to achieve the optimal control with regard to the just-in-time criterion. Proposition 3 implies that, for max-plus linear systems, the solution to the DDP by an output feedback control needs an infinite checking of the equivalence relations induced by the output mapping $C$ in equation (5). In order to avoid infinite checking for the solutions, the next subsection will present the $\gamma$-domain representation of max-plus linear systems and formulate DDP and MDDP in the $\gamma$-domain.

\section{$3.3 \gamma$-Domain Representation}

For a state equation in Eq. (3), each increasing sequence $\{x(k)\}$, it is possible to define the transformation $X(\gamma)=\bigoplus_{k \in \mathbb{Z}} x(k) \gamma^{k}$ where $\gamma$ is a backward shift operator in event domain (i.e., $Y(\gamma)=\gamma X(\gamma) \Longleftrightarrow\{y(k)\}=$ $\{x(k-1)\}$, (see [1], p. 228). This transformation is analogous to the $z$-transform used in discrete-time classical control theory and the formal series $X(\gamma)$ is a synthetic representation of the trajectory $x(k)$. The set of the formal power series in $\gamma$ is denoted by $\overline{\mathbb{Z}}_{\max } \llbracket \gamma \rrbracket$ and constitutes an idempotent semiring. Therefore, the state equation in Eq. (3) becomes a polynomial equation or an event-domain representation,

$X(\gamma)=\bar{A} X(\gamma) \oplus B U(\gamma) \oplus S Q(\gamma)$, where $\bar{A}=\gamma A$,

$Y(\gamma)=C X(\gamma)$

where the state $X(\gamma) \in\left(\overline{\mathbb{Z}}_{\max } \llbracket \gamma \rrbracket\right)^{n}$, the output $Y(\gamma) \in$ $\left(\overline{\mathbb{Z}}_{\max } \llbracket \gamma \rrbracket\right)^{q}$, the input $U(\gamma) \in\left(\overline{\mathbb{Z}}_{\max } \llbracket \gamma \rrbracket\right)^{p}$, and the disturbance $Q(\gamma) \in\left(\overline{\mathbb{Z}}_{\max } \llbracket \gamma \rrbracket\right)^{r}$, and matrices $\bar{A} \triangleq \gamma A \in$ $\left(\overline{\mathbb{Z}}_{\max } \llbracket \gamma \rrbracket\right)^{n \times n}, B \in\left(\overline{\mathbb{Z}}_{\max } \llbracket \gamma \rrbracket\right)^{n \times p}, C \in\left(\overline{\mathbb{Z}}_{\max } \llbracket \gamma \rrbracket\right)^{q \times n}$ and $S \in\left(\overline{\mathbb{Z}}_{\max } \llbracket \gamma \rrbracket\right)^{n \times r}$ represent the link between transitions. According to the state equation (6), the evolution of the system is

$$
\begin{aligned}
& X(\gamma)=\bar{A}^{*} B U(\gamma) \oplus \bar{A}^{*} S Q(\gamma) \\
& Y(\gamma)=C \bar{A}^{*} B U(\gamma) \oplus C \bar{A}^{*} S Q(\gamma)
\end{aligned}
$$

The trajectories $U(\gamma)$ and $Y(\gamma)$ can be related $([1]$, p. 243) by the equation $Y(\gamma)=H(\gamma) U(\gamma)$, where $H(\gamma)=$ $C \bar{A}^{*} B \in\left(\overline{\mathbb{Z}}_{\max } \llbracket \gamma \rrbracket\right)^{q \times p}$ is called the transfer matrix of the TEG. Entries of matrix $H$ are periodic series ([1], p. $260)$ in the idempotent semiring, usually represented by $p(\gamma) \oplus q(\gamma)\left(\tau \gamma^{\nu}\right)^{*}$, where $p(\gamma)$ is a polynomial representing the transient behavior, $q(\gamma)$ is a polynomial corresponding to a pattern which is repeated periodically, the period being given by the monomial $\left(\tau \gamma^{\nu}\right)$. The disturbances are uncontrollable inputs acting on the system internal's state, which model events that block the system, e.g. machine breakdown, uncontrollable component supply through matrix $S$, and $C \bar{A}^{*} S \in\left(\overline{\mathbb{Z}}_{\max } \llbracket \gamma \rrbracket\right)^{q \times r}$ is the transfer function between the disturbances and outputs.

\section{Solving DDP and MDDP by an Integrated Control Strategy}

\subsection{Solving DDP and MDDP by Open-loop Controls}

The objective of the MDDP is to find the greatest openloop or output feedback control $U(\gamma)$ such that the output trajectories will not be delayed more than the disturbance signals have acted on the system. For example, if a manufacturing system encounters a service breakdown, the control $U(\gamma)$ will delay the input of parts as much as possible to avoid congestion inside the system, while the system outputs will remain the same as before. Formally, according to Definition 6, this means to find the greatest control, $U(\gamma)$, such that the following 
equation holds,

$$
\begin{aligned}
& C \bar{A}^{*} B U(\gamma) \oplus C \bar{A}^{*} S Q(\gamma)=C \bar{A}^{*} S Q(\gamma) \\
& \Longleftrightarrow C \bar{A}^{*} B U(\gamma) \preceq C \bar{A}^{*} S Q(\gamma) .
\end{aligned}
$$

If the control $U(\gamma)=P Q(\gamma)$, where $P$ is a prefilter which generates the control by taking the disturbances into account, then, solving the MDDP is equivalent as finding a prefilter $P$ satisfying

$C \bar{A}^{*} B P Q(\gamma) \oplus C \bar{A}^{*} S Q(\gamma)=C \bar{A}^{*} S Q(\gamma), \forall Q(\gamma)$.

For any external input $V(\gamma)$, if $U(\gamma)=P Q(\gamma) \oplus V(\gamma)$, the equality above is equivalent as

$$
\begin{aligned}
& C \bar{A}^{*} B P Q(\gamma) \oplus C \bar{A}^{*} B V(\gamma) \oplus C \bar{A}^{*} S Q(\gamma) \\
= & C \bar{A}^{*} S Q(\gamma) \oplus C \bar{A}^{*} B V(\gamma), \forall Q(\gamma), \forall V(\gamma) .
\end{aligned}
$$

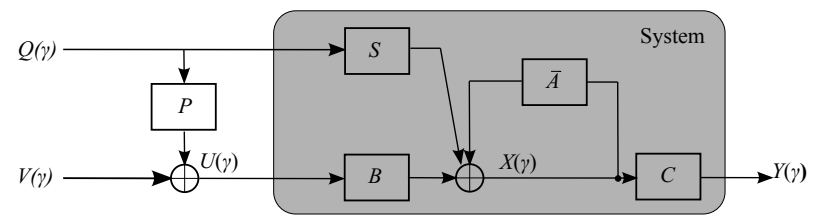

Fig. 1. The open-loop controller structure solving for DDP and MDDP.

Without loss of generality, we can consider a control strategy as depicted in Fig. 1, such that, for any initial condition $x_{0}$ in the state space, the system outputs induced by the control will not be disturbed more than the output signals delayed by the disturbance signals. In order to achieve Eq. (10), it is equivalent as finding $P$ satisfying

$$
\begin{aligned}
& C \bar{A}^{*} B P Q(\gamma) \preceq C \bar{A}^{*} S Q(\gamma), \forall Q(\gamma), \\
\Longleftrightarrow & C \bar{A}^{*} B P \preceq C \bar{A}^{*} S, \\
\Longleftrightarrow & P \preceq\left(C \bar{A}^{*} B\right) \phi\left(C \bar{A}^{*} S\right) \\
= & \bigoplus_{P \in \overline{\mathbb{Z}}_{\max } \llbracket \gamma \rrbracket^{p \times r}}\left\{C \bar{A}^{*} B P \preceq C \bar{A}^{*} S\right\} \triangleq P_{o p t} .
\end{aligned}
$$

Therefore, $P_{\text {opt }}$ is an optimal mapping that is independent of any disturbance $Q(\gamma)$, and leads to the optimal control $U_{o p t}(\gamma)=P_{o p t} Q(\gamma)$ which solves the MDDP for any disturbance $Q(\gamma)$. This optimal mapping is worth of interests since $U_{\text {opt }}(\gamma)$ is the greatest control delaying as much as possible the inputs in the system and thus reducing optimally useless waiting time.

\subsection{Relationship between $P_{\text {opt }}$ and DDP}

The MDDP is always solvable because the control signals, in the worst case, can be chosen to be $\varepsilon$ to achieve the solvability. Our interest is whether a link can be established between the solutions to the MDDP and the solutions to the DDP of max-plus linear systems. The question is whether the optimal solution $P_{o p t} Q(\gamma)$ will be able to solve the DDP of max-plus linear systems. According to Definition 5, solving the DDP in eventdomain means that the control $U(\gamma)$ has to achieve

$$
\begin{array}{r}
C \bar{A}^{*} B U(\gamma) \oplus C \bar{A}^{*} S Q(\gamma)=C \bar{A}^{*} B U(\gamma) \\
\Longleftrightarrow C \bar{A}^{*} S Q(\gamma) \preceq C \bar{A}^{*} B U(\gamma) .
\end{array}
$$

If $U(\gamma)=P Q(\gamma) \oplus V(\gamma)$ for any external input $V(\gamma)$ and disturbance $Q(\gamma)$, the inequality above is equivalent to

$C \bar{A}^{*} S Q(\gamma) \preceq C \bar{A}^{*} B P Q(\gamma) \oplus C \bar{A}^{*} B V(\gamma)$.

We can have the following necessary and sufficient condition for the optimal controller $U_{o p t}(\gamma)=P_{o p t} Q(\gamma) \oplus$ $V(\gamma)$, for any $Q(\gamma)$ and $V(\gamma)$, solving the MDDP to solve the DDP as well.

Theorem 4 ([25]) The optimal control $U_{\text {opt }}(\gamma)=$ $P_{\text {opt }} Q(\gamma) \oplus V(\gamma)$ to the MDDP also solves the DDP for the max-plus linear systems described in Eq. (6), where $P_{o p t}=\left(C \bar{A}^{*} B\right) \phi\left(C \bar{A}^{*} S\right)$, and for any disturbances $Q(\gamma)$ and external inputs $V(\gamma)$, if and only if Im $C \bar{A}^{*} S \subset \operatorname{Im} C \bar{A}^{*} B$.

Proof: "£ Sufficiency": If the condition $\operatorname{Im} C \bar{A}^{*} S \subset$ Im $C \bar{A}^{*} B$ holds, i.e., if $\exists L$ such that $C \bar{A}^{*} S=C \bar{A}^{*} B L$ then the optimal mapping $P_{\text {opt }}$ satisfies the following equality due to Eq. (f.8) in the Appendix,

$$
\begin{aligned}
& C \bar{A}^{*} B P_{o p t}=C \bar{A}^{*} B\left(\left(C \bar{A}^{*} B\right) \phi\left(C \bar{A}^{*} B L\right)\right) \\
& =C \bar{A}^{*} B L=C \bar{A}^{*} S,
\end{aligned}
$$

which implies

$$
\begin{aligned}
& C \bar{A}^{*} B P_{o p t} Q(\gamma) \oplus C \bar{A}^{*} S Q(\gamma) \oplus C \bar{A}^{*} B V(\gamma) \\
& =C \bar{A}^{*} B P_{o p t} Q(\gamma) \oplus C \bar{A}^{*} B V(\gamma)=C \bar{A}^{*} B U_{o p t}(\gamma) .
\end{aligned}
$$

Hence, the equality in (17) is equivalent as

$$
\begin{aligned}
& C \bar{A}^{*} B U_{\text {opt }}(\gamma) \oplus C \bar{A}^{*} S Q(\gamma)=C \bar{A}^{*} B U_{\text {opt }}(\gamma) \\
& \Leftrightarrow C \bar{A}^{*} S Q(\gamma) \preceq C \bar{A}^{*} B U_{\text {opt }}(\gamma) .
\end{aligned}
$$

Eq. (18) means that the same optimal control $U_{\text {opt }}(\gamma)=$ $P_{\text {opt }} Q(\gamma) \oplus V(\gamma)$ can also solve the DDP in Definition 5 for any given disturbance $Q(\gamma)$ and external input $V(\gamma)$.

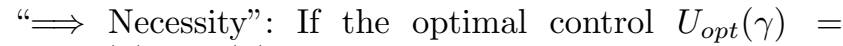
$P_{\text {opt }} Q(\gamma) \oplus V(\gamma)$ provides the optimal solution to the MDDP for any disturbance signal $Q(\gamma)$ and any choice of the external input $V(\gamma)$, and such a solution solves 
the DDP as well, then it implies that

$$
\begin{aligned}
& C \bar{A}^{*} B U_{o p t}(\gamma) \oplus C \bar{A}^{*} S Q(\gamma)=C \bar{A}^{*} B U_{o p t}(\gamma) \\
\Leftrightarrow & C \bar{A}^{*} B\left(P_{o p t} Q(\gamma) \oplus V(\gamma)\right) \oplus C \bar{A}^{*} S Q(\gamma) \\
& =C \bar{A}^{*} B\left(P_{o p t} Q(\gamma) \oplus V(\gamma)\right), \forall Q(\gamma), V(\gamma) \\
\Leftrightarrow & C \bar{A}^{*} B P_{o p t} Q(\gamma) \oplus C \bar{A}^{*} S Q(\gamma)=C \bar{A}^{*} B P_{o p t} Q(\gamma), \forall Q(\gamma) \\
\Leftrightarrow & C \bar{A}^{*} S Q(\gamma) \preceq C \bar{A}^{*} B P_{o p t} Q(\gamma), \forall Q(\gamma) .
\end{aligned}
$$

By definition $C \bar{A}^{*} B P_{o p t}=C \bar{A}^{*} B\left(\left(C \bar{A}^{*} B\right) \phi\left(C \bar{A}^{*} S\right)\right) \preceq$ $C \bar{A}^{*} S$, see Eq. (f.6) in the Appendix, then

$$
C \bar{A}^{*} B P_{o p t} Q(\gamma)=C \bar{A}^{*} S Q(\gamma)
$$

Therefore, we have $\operatorname{Im} C \bar{A}^{*} S \subset \operatorname{Im} C \bar{A}^{*} B$.

\subsection{Solving DDP and MDDP by Integrating Output} Feedback with Open-loop Control Strategies

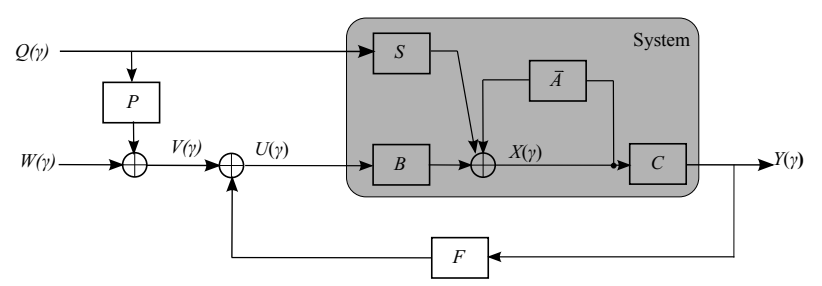

Fig. 2. The integrated controller structure solving for DDP and MDDP.

In this paper, the disturbance is assumed to be measurable and the control architecture is given in Fig. 2. We like to fix the external input $V(\gamma)$ such that $V(\gamma)=$ $P Q(\gamma) \oplus W(\gamma)$, for any disturbance $Q(\gamma)$ and external input $W(\gamma)$. The pre-filter matrix $P$ is assumed to be known and such that $P \preceq P_{o p t}$, where $P_{o p t}=$ $\left(C \bar{A}^{*} B\right) \phi\left(C \bar{A}^{*} S\right)$. First of all, we are looking for the output feedback matrix $F$ such that it would preserve the open-loop behaviors. Then we will give a necessary and sufficient condition such that this control architecture solves the DDP as well.

Mathematically, by solving the implicit equation in Eq. (6), the states and outputs in the $\gamma$-domain are represented as follows, where the output feedback controller $U(\gamma)=F Y(\gamma) \oplus V(\gamma)$, and $V(\gamma)=P Q(\gamma) \oplus W(\gamma)$ for any disturbance $Q(\gamma)$ and any external input $W(\gamma)$,

$$
\begin{aligned}
X(\gamma) & =(\bar{A} \oplus B F C)^{*} B V(\gamma) \oplus(\bar{A} \oplus B F C)^{*} S Q(\gamma) \\
& =(\bar{A} \oplus B F C)^{*}[B \mid S]\left(\begin{array}{l}
V(\gamma) \\
Q(\gamma)
\end{array}\right) \\
& =(\bar{A} \oplus B F C)^{*} \widetilde{B}\left(\begin{array}{c}
V(\gamma) \\
Q(\gamma)
\end{array}\right), \text { where } \widetilde{B}=[B \mid S] \\
Y(\gamma) & =C X(\gamma)=C(\bar{A} \oplus B F C)^{*} \widetilde{B}\left(\begin{array}{c}
V(\gamma) \\
Q(\gamma)
\end{array}\right)
\end{aligned}
$$

Based on Definition 5, solving the DDP in $\gamma$-domain means that the output feedback controller $U(\gamma)=$ $F Y(\gamma) \oplus V(\gamma)$ has to achieve the following equality:

$C(\bar{A} \oplus B F C)^{*} \widetilde{B}\left(\begin{array}{l}V(\gamma) \\ Q(\gamma)\end{array}\right)=C(\bar{A} \oplus B F C)^{*} B V(\gamma)$

Based on Definition 6, solving the MDDP in $\gamma$-domain means that the output feedback controller has to achieve another equality:

$C(\bar{A} \oplus B F C)^{*} \widetilde{B}\left(\begin{array}{l}V(\gamma) \\ Q(\gamma)\end{array}\right)=C(\bar{A} \oplus B F C)^{*} S Q(\gamma)$

Equations (20) and (21) each have three variables, the output feedback structure $F$, the open-loop controller $V(\gamma)$, as well as the disturbance input $Q(\gamma)$. If we need the pair of $F$ and $V(\gamma)$ to solve the MDDP and the DDP for any arbitrary disturbances, then we can proceed by fixing the open-loop control first and then find the output feedback control, or vice versa.

First, the goal is to find the integrated control law $U(\gamma)=F Y(\gamma) \oplus V(\gamma)$, such that the output signals are the same as the output signals controlled by any openloop controller $V(\gamma)$. In summary, that is, the following equality holds

$C(\bar{A} \oplus B F C)^{*} \widetilde{B}\left(\begin{array}{l}V(\gamma) \\ Q(\gamma)\end{array}\right)=C \bar{A}^{*} \widetilde{B}\left(\begin{array}{l}V(\gamma) \\ Q(\gamma)\end{array}\right)$

Proposition 5 ([16, 18]) The greatest output feedback controller $F_{\text {opt }}$ is given by

$$
F_{o p t}=\left(C \bar{A}^{*} B\right) \phi\left(C \bar{A}^{*} \widetilde{B}\right) \phi\left(C \bar{A}^{*} \widetilde{B}\right)
$$

such that the output trajectories generated by the output feedback controller are the same as the output trajectories 
generated by the open-loop controller, i.e. the equality $C\left(\bar{A} \oplus B F_{\text {opt }} C\right)^{*} \widetilde{B}=C \bar{A}^{*} \widetilde{B}$ holds.

Proposition 6 The integrated control law $U_{\text {opt }}(\gamma)=$ $F_{\text {opt }} Y(\gamma) \oplus P_{\text {opt }} Q(\gamma) \oplus W(\gamma), \forall Q(\gamma), \forall W(\gamma)$, solves the $M D D P$ of the max-plus linear system in Eq. (6), where $P_{\text {opt }}=\left(C \bar{A}^{*} B\right) \phi\left(C \bar{A}^{*} S\right)$ and the output feedback control matrix is $F_{\text {opt }}=\left(C \bar{A}^{*} B\right) \phi\left(C \bar{A}^{*} \widetilde{B}\right) \phi\left(C \bar{A}^{*} \widetilde{B}\right)$.

Proof: If we apply the integrated control law $U_{\text {opt }}(\gamma)=$ $F_{\text {opt }} Y(\gamma) \oplus P_{\text {opt }} Q(\gamma) \oplus W(\gamma)$ to the max-plus linear system in Eq. (6), then by Proposition 5, the following inequalities hold:

$$
\begin{aligned}
& C\left(\bar{A} \oplus B F_{o p t} C\right)^{*} \widetilde{B}\left(\begin{array}{c}
P_{o p t} Q(\gamma) \oplus W(\gamma) \\
Q(\gamma)
\end{array}\right) \\
= & C \bar{A}^{*} P_{o p t} Q(\gamma) \oplus C \bar{A}^{*} B W(\gamma) \oplus C \bar{A}^{*} S Q(\gamma),
\end{aligned}
$$

i.e. the open-loop behaviors are preserved. Moreover, due to the definition of $P_{\text {opt }}=\left(C \bar{A}^{*} B\right) \phi\left(C \bar{A}^{*} S\right)$, then Eq. (24) is less than

$$
\begin{aligned}
& C \bar{A}^{*} B W(\gamma) \oplus C \bar{A}^{*} S Q(\gamma) \\
\preceq & C\left(\bar{A} \oplus B F_{\text {opt }} C\right)^{*} S Q(\gamma) \oplus C\left(\bar{A} \oplus B F_{\text {opt }} C\right)^{*} B W(\gamma) .
\end{aligned}
$$

Clearly, the last term is less than Eq. (24), Hence, we can achieve the following equality

$$
\begin{array}{r}
C\left(\bar{A} \oplus B F_{\text {opt }} C\right)^{*} \widetilde{B}\left(\begin{array}{c}
P_{o p t} Q(\gamma) \oplus W(\gamma) \\
Q(\gamma)
\end{array}\right) \\
=C\left(\bar{A} \oplus B F_{o p t} C\right)^{*} \widetilde{B}\left(\begin{array}{c}
W(\gamma) \\
Q(\gamma)
\end{array}\right), \forall Q(\gamma), W(\gamma) .
\end{array}
$$

Because $W(\gamma)$ can be arbitrary, without loss of generality, for $W(\gamma)=\varepsilon$, the equality above is equivalent to the following equality

$$
\begin{array}{r}
C\left(\bar{A} \oplus B F_{o p t} C\right)^{*} B P_{o p t} Q(\gamma) \oplus C\left(\bar{A} \oplus B F_{o p t} C\right)^{*} S Q(\gamma) \\
=C\left(\bar{A} \oplus B F_{o p t} C\right)^{*} S Q(\gamma), \forall Q(\gamma) .
\end{array}
$$

Hence, the integrated control $U_{\text {opt }}(\gamma)=F_{\text {opt }} Y(\gamma) \oplus$ $P_{\text {opt }} Q(\gamma)$ solves the MDDP. Because $W(\gamma)$ can be an arbitrary input, the integrated control $U_{\text {opt }}(\gamma)=$ $F_{\text {opt }} Y(\gamma) \oplus P_{\text {opt }} Q(\gamma) \oplus W(\gamma)$ can solve the MDDP as well.

Proposition 7 The integrated control law $U_{\text {opt }}(\gamma)=$ $F_{\text {opt }} Y(\gamma) \oplus P_{o p t} Q(\gamma) \oplus W(\gamma), \forall Q(\gamma), \forall W(\gamma)$, solves the $D D P$ of the max-plus linear system in Eq. (6) if and only if $\operatorname{Im} C \bar{A}^{*} S \subset \operatorname{Im} C \bar{A}^{*} B$, where the open-loop control is
$P_{\text {opt }}=\left(C \bar{A}^{*} B\right) \phi\left(C \bar{A}^{*} S\right)$ and the output feedback control matrix is $F_{\text {opt }}=\left(C \bar{A}^{*} B\right) \phi\left(C \bar{A}^{*} \widetilde{B}\right) \phi\left(C \bar{A}^{*} \widetilde{B}\right)$.

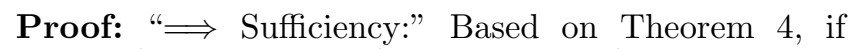
Im $C \bar{A}^{*} S \subset \operatorname{Im} C \bar{A}^{*} B$, then $C \bar{A}^{*} B P_{\text {opt }} Q(\gamma)=$ $C \bar{A}^{*} S Q(\gamma)$ for any $Q(\gamma)$. In other words, Eq. (22) becomes

$$
\begin{aligned}
& C\left(\bar{A} \oplus B F_{o p t} C\right)^{*} \widetilde{B}\left(\begin{array}{c}
P_{o p t} Q(\gamma) \oplus W(\gamma) \\
Q(\gamma)
\end{array}\right) \\
= & C \bar{A}^{*} B P_{o p t} Q(\gamma) \oplus C \bar{A}^{*} B W(\gamma) \oplus C \bar{A}^{*} S Q(\gamma), \\
= & C \bar{A}^{*} B P_{o p t} Q(\gamma) \oplus C \bar{A}^{*} B W(\gamma)=C \bar{A}^{*} B V(\gamma) \\
= & C\left(\bar{A} \oplus B F_{\text {opt }} C\right)^{*} B V(\gamma), \\
& \text { where } V(\gamma)=P_{\text {opt }} Q(\gamma) \oplus W(\gamma) .
\end{aligned}
$$

Hence, we can achieve the following equality

$$
\begin{array}{r}
C\left(\bar{A} \oplus B F_{o p t} C\right)^{*} B V(\gamma) \oplus C\left(\bar{A} \oplus B F_{o p t} C\right)^{*} S Q(\gamma) \\
=C\left(\bar{A} \oplus B F_{o p t} C\right)^{*} B V(\gamma) .
\end{array}
$$

Therefore, such an integrated control $U_{\text {opt }}(\gamma)$ solves the DDP.

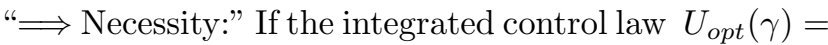
$F_{\text {opt }} Y(\gamma) \oplus P_{\text {opt }} Q(\gamma) \oplus W(\gamma)$ solves the DDP, then the following equality holds

$$
\begin{array}{r}
C\left(\bar{A} \oplus B F_{o p t} C\right)^{*} B V(\gamma) \oplus C\left(\bar{A} \oplus B F_{\text {opt }} C\right)^{*} S Q(\gamma) \\
=C\left(\bar{A} \oplus B F_{\text {opt }} C\right)^{*} B V(\gamma),
\end{array}
$$

for $V(\gamma)=P_{o p t} Q(\gamma) \oplus W(\gamma), \forall Q(\gamma)$ and $\forall W(\gamma)$. Because the feedback control $F_{\text {opt }}$ preserves the open-loop behaviors, the equality above implies

$C \bar{A}^{*} B V(\gamma) \oplus C \bar{A}^{*} S Q(\gamma)=C \bar{A}^{*} B V(\gamma)$,

for $V(\gamma)=P_{o p t} Q(\gamma) \oplus W(\gamma)$ with an arbitrary $W(\gamma)$. Without loss of generality, for $W(\gamma)=\varepsilon$, the equality above is equivalent to

$$
C \bar{A}^{*} B P_{o p t} Q(\gamma) \oplus C \bar{A}^{*} S Q(\gamma)=C \bar{A}^{*} B P_{o p t} Q(\gamma) .
$$

Based on Theorem 4, we have $\operatorname{Im} C \bar{A}^{*} S \subset \operatorname{Im} C \bar{A}^{*} B$.

\section{Evaluating the Distances between Solutions to DDP and MDDP in the Event Domain}

If the condition $\operatorname{Im} C \bar{A}^{*} S \subset \operatorname{Im} C \bar{A}^{*} B$ in Theorem 4 is not satisfied, we can study the differences between the output trajectories solving for the MDDP and the output trajectories solving for the DDP. The difference will give us an upper bound or an evaluation of how much more delays or tokens needed for the control to the MDDP in order to solve the DDP. 
Definition 7 ([22]) Let $X(\gamma)$ and $Y(\gamma)$ be two vector $\gamma$-series with dimensions $n$ and $q$ associated to dater function $k \mapsto x(k)$ and $k \mapsto y(k)$. The residuation $X(\gamma) \phi Y(\gamma)$ is named as the correlation matrix of $X(\gamma)$ over $Y(\gamma)$. If $X(\gamma)=Y(\gamma)$, then the residuation $X(\gamma) \phi Y(\gamma)$ is called the autocorrelation matrix of $X(\gamma)$.

The residuation $X(\gamma) \phi Y(\gamma)$ allows us to evaluate the distances between trajectories $X(\gamma)$ and $Y(\gamma)$ in the event domain, or in the time domain if we consider the second order theory in $([22])$.

Definition 8 ([24]) Let $x(\gamma)=\oplus_{k \in \mathbb{Z}} x(k) \gamma^{k}$ and $y(\gamma)=$ $\oplus_{k \in \mathbb{Z}} y(k) \gamma^{k}$ be two scalar $\gamma$-series associated to dater function $k \mapsto x(k)$ and $k \mapsto y(k)$. We denote $\mathcal{C}_{x}(t)$ and $\mathcal{C}_{y}(t)$ as the counter function associated to $x(\gamma)$ and $y(\gamma)$, i.e. $x(\gamma)=\oplus_{t \in \mathbb{Z}} t \gamma^{\mathcal{C}_{x}(t)}$, and $y(\gamma)=\oplus_{t \in \mathbb{Z}} t \gamma^{\mathcal{C}_{y}(t)}$. Distance in the event domain is defined by

$$
\begin{aligned}
\Delta_{X Y} & =\max \left\{\left|\mathcal{C}_{x}(t)-\mathcal{C}_{y}(t)\right| \text { for all } t \in \mathbb{Z}\right\} \\
& =\mathcal{C}_{(x \wedge y) \phi(x \oplus y)}(0) .
\end{aligned}
$$

The last term $\mathcal{C}_{(x \wedge y) \phi(x \oplus y)}(0)$ is the tightest constant upper bound for the tokens in the timed event graphs.

Remark 1 Given two pairs $(x, y)$ and $\left(x^{\prime}, y^{\prime}\right)$ of scalar $\gamma$-series satisfying $(x \wedge y) \phi(x \oplus y) \succeq\left(x^{\prime} \wedge y^{\prime}\right) \phi\left(X^{\prime} \oplus Y^{\prime}\right)$, then $\mathcal{C}_{(x \wedge y) \phi(x \oplus y)}(0) \leq \mathcal{C}_{\left(x^{\prime} \wedge y^{\prime}\right) \phi\left(X^{\prime} \oplus Y^{\prime}\right)}(0)$, i.e.

$(x \wedge y) \phi(x \oplus y) \succeq\left(x^{\prime} \wedge y^{\prime}\right) \phi\left(x^{\prime} \oplus y^{\prime}\right) \Longleftrightarrow \Delta_{x y} \leq \Delta_{x^{\prime} y^{\prime}}$

Remark 2 If two scalar $\gamma$ series $x(\gamma)$ and $y(\gamma)$ satisfy $x(\gamma) \preceq y(\gamma)$, then the distance in the event domain $\Delta_{x y}$ can be written as $\Delta_{x y}=\mathcal{C}_{x \phi y}(0)$. Moreover, if $\Delta_{x y} \leq M$, where $M$ is a finite bound, then the following inequalities are satisfied:

$$
\begin{aligned}
& x \phi y \succeq \gamma^{M} \Longrightarrow x \succeq(x \phi y) y \succeq \gamma^{M} y, \\
& \text { or, } x(k) \succeq y(k-M), \forall k .
\end{aligned}
$$

Because $\gamma$ is used to denote the token in TEG, the multiplier $M$ denotes how many tokens in the places between two transitions. These inequalities above can describe an evaluation on how many tokens or stocks between the two transitions are needed in timed event graphs.

Proposition 8 Let $x_{1}(\gamma)=s_{1}(\gamma) u(\gamma)$ and $x_{2}(\gamma)=$ $s_{2}(\gamma) u(\gamma)$ two scalar $\gamma$-series describing the behaviors of two states for max-plus linear systems. The distance between these two trajectories in the event domain is bounded by the distance between the two transfer function series, that is,

$$
\begin{aligned}
\Delta_{x_{1} x_{2}} & =\mathcal{C}_{\left(x_{1} \wedge x_{2}\right) \phi\left(x_{1} \oplus x_{2}\right)}(0) \\
& \leq \Delta_{s_{1} s_{2}}=\mathcal{C}_{\left(s_{1} \wedge s_{2}\right) \phi\left(s_{1} \oplus s_{2}\right)}(0),
\end{aligned}
$$

for any input $u(\gamma)$.
Proof: Based on the residuation theory in [1] as well as Appendix, we have the following derivations:

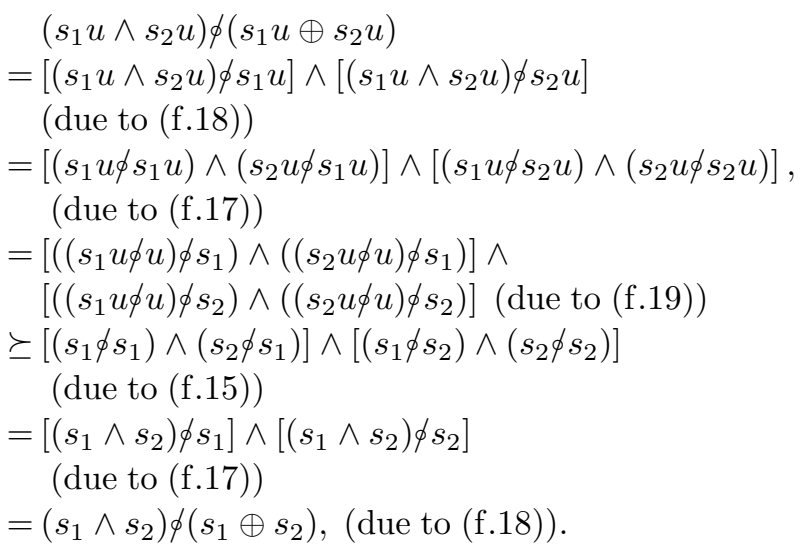

Hence, $\forall u(\gamma)$, the following inequality holds:

$$
\left(s_{1} u \wedge s_{2} u\right) \phi\left(s_{1} u \oplus s_{2} u\right) \succeq\left(s_{1} \wedge s_{2}\right) \phi\left(s_{1} \oplus s_{2}\right),
$$

which implies directly:

$$
\mathcal{C}_{\left(x_{1} \wedge x_{2}\right) \phi\left(x_{1} \oplus x_{2}\right)}(0) \leq \mathcal{C}_{\left(s_{1} \wedge s_{2}\right) \phi\left(s_{1} \oplus s_{2}\right)}(0),
$$

for any input $u(\gamma)$.

Remark 3 If $s_{1}(\gamma) \preceq s_{2}(\gamma)$, then $\left(s_{1} \wedge s_{2}\right) \phi\left(s_{1} \oplus s_{2}\right)=$ $s_{1} \phi s_{2}$.

Theorem 9 ([22] Increasing Correlation Principle) Let $X_{1}(\gamma)=S(\gamma) U(\gamma)$ and $X_{2}(\gamma)=S(\gamma) V(\gamma)$ be the output vectors corresponding to input vectors $U(\gamma)$ and $V(\gamma)$, then

$X_{1} \phi X_{2} \succeq(V \phi U)(S \phi S)$,

$X_{1} \phi X_{2} \succeq(U \phi V) \operatorname{Tr}^{\wedge}(S \phi S)$,

where for any matrix $A$, the dual trace $\operatorname{Tr}^{\wedge}(A)$ is defined as $\operatorname{Tr}^{\wedge}(A)=\bigwedge_{i}(A)_{i i}$.

We can use the Increasing Correlation Principle to generate the preceding results to matrix forms of transfer series.

Theorem 10 Let $X_{1}(\gamma)=S_{1}(\gamma) U(\gamma)$ and $X_{2}(\gamma)=$ $S_{2}(\gamma) U(\gamma)$ be the output vectors corresponding to the same input vector $U(\gamma)$, then

$X_{1} \phi X_{2} \succeq S_{1} \phi S_{2}$,

$X_{1} \phi X_{2} \succeq \operatorname{Tr}^{\wedge}\left(S_{2} \phi S_{1}\right)$,

where $\operatorname{Tr}^{\wedge}\left(S_{2} \phi S_{1}\right)=\bigwedge_{i}\left(S_{2} \phi S_{1}\right)_{i i}$. 
Proof: Based on the residuation theory in ([1]), we have the following derivations:

$$
\begin{aligned}
X_{1} \phi X_{2} & =\left(S_{1} U\right) \phi\left(S_{2} U\right) \\
& =\left(\left(S_{1} U\right) \phi U\right) \phi S_{2}, \text { due to (f.19) } \\
& \succeq S_{1} \phi S_{2}, \text { due to (f.15) }
\end{aligned}
$$

and

$$
\begin{aligned}
X_{1} \phi X_{2}= & \left(S_{1} U\right) \phi\left(S_{2} U\right) \\
= & U \phi\left(S_{1} \phi\left(S_{2} U\right)\right), \text { due to }(\mathrm{f} .11) \\
\succeq & U \phi\left(\left(S_{1} \phi S_{2}\right) U\right), \text { due to }(\mathrm{f} .13) \\
\succeq & U \phi\left[\left(\operatorname{Tr}^{\wedge}\left(S_{2} \phi S_{1}\right)\right) U\right], \\
& \quad \text { due to } \operatorname{Tr}^{\wedge}\left(S_{2} \phi S_{1}\right)=S_{1} \phi S_{2}, \\
\succeq & (U \phi U) \operatorname{Tr}^{\wedge}\left(S_{2} \phi S_{1}\right), \\
& \quad \text { due to } \operatorname{Tr}^{\wedge}\left(S_{2} \phi S_{1}\right) \text { is scalar, } \\
\succeq & \operatorname{Tr}^{\wedge}\left(S_{2} \phi S_{1}\right) \\
& \quad \text { due to } U \phi U \succeq \mathrm{Id} .
\end{aligned}
$$

In the remaining section, we will apply the preceding results in order to compare the difference between the output trajectories induced by controls solving for the DDP and the MDDP, i.e, the distance between $Y_{1}=$ $C \bar{A}^{*} B P_{o p t} Q(\gamma)$, and $Y_{2}=C \bar{A}^{*} S Q(\gamma)$. The two output trajectories are obviously ordered because $P_{\text {opt }}=$ $\left(C \bar{A}^{*} B\right) \phi\left(C \bar{A}^{*} S\right)$, i.e. $Y_{1} \preceq Y_{2}$, which solves for the MDDP. On the other hand if $Y_{1}=Y_{2}$, then the DDP is solved. The question is if $Y_{1} \prec Y_{2}$, then how much is the distance between the two output trajectories.

Proposition 11 Let $Y_{1}(\gamma)=C \bar{A}^{*} B P_{o p t} Q(\gamma)$ and $Y_{2}(\gamma)=C \bar{A}^{*} S Q(\gamma)$ two $\gamma$-series describing the output behaviors of the max-plus linear systems in Eq. (6), where $P_{\text {opt }}=\left(C \bar{A}^{*} B\right) \phi\left(C \bar{A}^{*} S\right)$. The distance between these two output trajectories in the event domain is bounded by the distance between the two transfer function series, that is,

$\Delta_{Y_{1} Y_{2}}=\mathcal{C}_{Y_{1} \phi Y_{2}}(0) \leq \mathcal{C}_{\left(C \bar{A}^{*} B P_{o p t}\right) \phi\left(C \bar{A}^{*} S\right)}(0)$,

for any distance input $Q(\gamma)$.

Proof: Using Eq. (29) in Proposition 10, we can obtain that $Y_{1} \phi Y_{2}=\left(C \bar{A}^{*} B P_{o p t} Q\right) \phi\left(C \bar{A}^{*} S Q\right) \succeq$ $\left(C \bar{A}^{*} B P_{\text {opt }}\right) \phi\left(C \bar{A}^{*} S\right)$. Therefore, the distance between these two output trajectories in the event domain satisfies the following equality

$$
\Delta_{Y_{1} Y_{2}}=\mathcal{C}_{Y_{1} \phi Y_{2}}(0) \leq \mathcal{C}_{\left(C \bar{A}^{*} B P_{\text {opt }}\right) \phi\left(C \bar{A}^{*} S\right)}(0)
$$

\section{Classes of Disturbances Ensuring Solvability of DDP}

If the condition $\operatorname{Im} C \bar{A}^{*} S \subset \operatorname{Im} C \bar{A}^{*} B$ in Theorem 4 is not satisfied, then the optimal solution
$P_{\text {opt }}=\left(C \bar{A}^{*} B\right) \phi\left(C \bar{A}^{*} S\right)$ to the MDDP will not be able to solve the DDP. Besides calculating the distance between these two solutions, another interesting question is to ask what type of disturbances will allow us to solve the DDP using the same optimal control $P_{o p t}$ of the MDDP. In other words, for any disturbance $Q(\gamma)$, we need to achieve the equality (17). Our proposed approach is that, instead of keeping the original matrix $S$ which will not solve the DDP by $P_{o p t}$, we can try to find conditions on the matrix $\widetilde{S}$, while keeping the control law $U(\gamma)=P_{o p t} Q(\gamma)$, such that the DDP be solved.

\section{Lemma 12 Matrix}

$\widetilde{S}_{\text {opt }} \triangleq\left(C \bar{A}^{*}\right) \phi\left(C \bar{A}^{*} B P_{\text {opt }}\right)$

achieves equality $C \bar{A}^{*} B P_{\text {opt }}=C \bar{A}^{*} \widetilde{S}_{\text {opt }}$.

Proof: First, according to the residuation definition, the matrix $\widetilde{S}_{o p t}$ is the greatest matrix such that $C \bar{A}^{*} \widetilde{S}_{o p t} \preceq$ $C \bar{A}^{*} B P_{\text {opt }}$. On the other hand, due to the property of $L_{a}^{\sharp} \circ L_{a} \succeq I d$, we have $\widetilde{S}_{\text {opt }}=\left(C \bar{A}^{*}\right) \phi\left(C \bar{A}^{*} B P_{\text {opt }}\right) \succeq$ $B P_{\text {opt }}$. This implies $C \bar{A}^{*} \widetilde{S}_{\text {opt }} \succeq C \bar{A}^{*} B P_{\text {opt }}$, for an isotone mapping $C \bar{A}^{*}$. Therefore, the following equality holds $C \bar{A}^{*} B P_{\text {opt }}=C \bar{A}^{*} \widetilde{S}_{\text {opt }}$.

Proposition 13 All matrix $\widetilde{S} \preceq \widetilde{S}_{o p t}$ is such that the open-loop control $U(\gamma)=P_{\text {opt }} Q \overline{(\gamma)}$ solves for the DDP, where $P_{\text {opt }}=\left(C \bar{A}^{*} B\right) \phi\left(C \bar{A}^{*} S\right)$.

Proof: From Lemma 12, it is clear that equality (17) is achieved, i.e., the DDP is solved with $\widetilde{S}_{o p t}$ and $P_{o p t}$, furthermore the following implication holds $\widetilde{S} \preceq \widetilde{S}_{\text {opt }} \Rightarrow$ $C \bar{A}^{*} \widetilde{S} \preceq C \bar{A}^{*} \widetilde{S}_{\text {opt }}=C \bar{A}^{*} B P_{\text {opt }}$, hence, equality (17) is achieved, i.e., the DDP is solvable by the optimal control $P_{\text {opt }}$ with any matrix $\widetilde{S} \preceq \widetilde{S}_{\text {opt }}$.

Proposition 14 Given the max-plus linear systems in Eq. (6), where $S$ is replaced by a new matrix $\widetilde{S}$ satisfying $\widetilde{S} \preceq \widetilde{S}_{o p t}=\left(C \bar{A}^{*}\right) \phi\left(C \bar{A}^{*} B P_{o p t}\right)$, and the optimal integrated control $U_{\text {opt }}(\gamma)=F_{\text {opt }}^{\prime} Y(\gamma) \oplus P_{\text {opt }} Q(\gamma) \oplus W(\gamma)$ solves for the MDDP and the DDP simultaneously, where $P_{\text {opt }}=\left(C \bar{A}^{*} B\right) \phi\left(C \bar{A}^{*} S\right)$, the feedback control $F_{\text {opt }}^{\prime}$ given by

$$
F_{o p t}^{\prime}=\left(C \bar{A}^{*} B\right) \phi\left(C \bar{A}^{*} \widetilde{B}\right) \phi\left(C \bar{A}^{*} \widetilde{B}\right) .
$$

Proof: The proof is a direct extension of the previous propositions, the feedback structure $F_{o p t}^{\prime}=$ $\left(C \bar{A}^{*} B\right) \phi\left(C \bar{A}^{*} \widetilde{B}\right) \phi\left(\bar{A}^{*} \widetilde{B}\right)$ preserves the openloop behaviors induced by the open-loop control $P_{\text {opt }} Q(\gamma) \oplus W(\gamma)$. Such an open-loop control solves for 
the DDP and MDDP, and it ensures that the integration of the feedback control $F_{o p t}^{\prime}$ and the open-loop control $U(\gamma)$ solves the DDP and MDDP as well.

\section{Application to a High Throughput Screening System in Drug Discovery}

High throughput screening (HTS) is a standard technology in drug discovery. In HTS systems, optimal scheduling is required to finish the screening in the shortest time, as well as to preserve the consistent time spending on each activity. The HTS system in this section, adapted from [3], consists of three activities: activity 1 , executed on the resource Pipettor, is filling the chemical compound A into the wells of a microplate, which lasts for 3 time units. Next, the microplate is transferred to a storage or incubator for at least 1 time unit. Before the waiting time is done for the compound A, activity 3 , executed on the resource Robot, is that the robot picks up another compound B, which takes 1 time unit. Once the 1 unit waiting time for the compound $\mathrm{A}$ is over, the robot moves the compound $\mathrm{B}$ to the microplate carrying the substance A. Activity 2 is that the microplate containing the compound $\mathrm{AB}$ is transferred to the pipettor again for 4 time units.

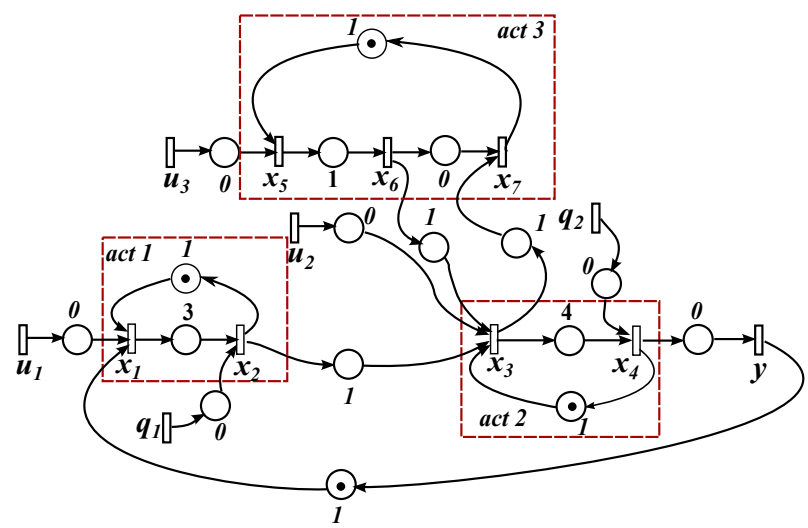

Fig. 3. The time event graph model of the HTS system.

If we are interested in the start and release event time of each activity, we can model the HTS system as a TEG model, shown in Fig. 3, in which $x_{1}$ and $x_{2}$ denote the start and release time of the activity 1 on the pipettor, denoted as act 1, $x_{3}$ and $x_{4}$ denote the start and release time of the activity 2 on the same pipettor, denoted as act 2 , and $x_{5}$ and $x_{7}$ denote the start and release time of the activity 3 on the robot, denoted as act 3. $x_{6}$ is the event time that the compound $\mathrm{B}$ is ready to be transferred to the microplate containing the compound $\mathrm{A}$. The inputs $u_{1}, u_{2}$, and $u_{3}$, are the starting time of the activity 1 , 2 , and 3 , respectively, which the users can decide when to load the chemical compounds. The disturbance $q_{1}$ delays the release time of the pipettor after activity 1 , and the disturbance $q_{2}$ delays the release time of the pipettor after activity 2 , e.g. uncontrollable system delays or uncontrollable transition time delays. The output $y$ is the release time of the pipettor. The circles represent places and the bars represent the transitions $x_{i}$. Each black token in the places represents that the corresponding resource is available, i.e. the activity is ready to start.

For the TEG model of a HTS system shown in Fig. 3, the implicit equation in $\mathbb{Z}_{\max }$ can be written as

$$
\begin{aligned}
& x(k)=A_{0} x(k) \oplus A_{1} x(k-1) \oplus B u(k) \oplus S q(k), \\
& y(k)=C x(k),
\end{aligned}
$$

where

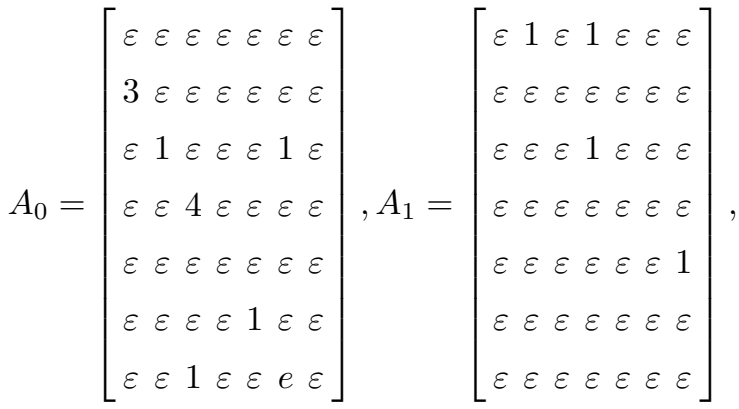

$$
\begin{aligned}
& B=\left[\begin{array}{ccc}
e & \varepsilon & \varepsilon \\
\varepsilon & \varepsilon & \varepsilon \\
\varepsilon & e & \varepsilon \\
\varepsilon & \varepsilon & \varepsilon \\
\varepsilon & \varepsilon & e \\
\varepsilon & \varepsilon & \varepsilon \\
\varepsilon & \varepsilon & \varepsilon
\end{array}\right], S=\left[\begin{array}{cc}
\varepsilon & \varepsilon \\
e & \varepsilon \\
\varepsilon & \varepsilon \\
\varepsilon & e \\
\varepsilon & \varepsilon \\
\varepsilon & \varepsilon \\
\varepsilon & \varepsilon
\end{array}\right], \\
& C=\left[\begin{array}{lllllll}
\varepsilon & \varepsilon & \varepsilon & e & \varepsilon & \varepsilon & \varepsilon
\end{array}\right] \text {. }
\end{aligned}
$$

Moreover, the system over the max-plus algebra $\overline{\mathbb{Z}}_{\max } \llbracket \gamma \rrbracket$ in $\gamma$-domain is described as the following:

$$
\begin{aligned}
& X(\gamma)=\bar{A} X(\gamma) \oplus B U(\gamma) \oplus S Q(\gamma), \text { with } \bar{A}=A_{0} \oplus \gamma A_{1}, \\
& Y(\gamma)=C X(\gamma) .
\end{aligned}
$$

The example has been computed by using the toolbox MinMaxGD, a $\mathrm{C}++$ library allowing to handle periodic series as introduced in ([9]), and it can be noted that this library is also interfaced with Scilab and MATLAB. We obtain the transfer functions between the input $U(\gamma)$ and the output $Y(\gamma)$, and between the disturbance $Q(\gamma)$ and the output $Y(\gamma)$, are given, respectively, as

$$
\begin{aligned}
C \bar{A}^{*} B & =\left[8(9 \gamma)^{*} 4(9 \gamma)^{*} 6(9 \gamma)^{*}\right], \\
C \bar{A}^{*} S & =\left[5(9 \gamma)^{*}(9 \gamma)^{*}\right],
\end{aligned}
$$

in which each component of these matrices consists of periodic series. Essentially, the $\gamma$-periodic series represent the output sequence when an infinity of tokens is 
put in the system at time 0 (impulse input). For instance, $C \bar{A}^{*} B(1,1)=8(9 \gamma) *$ represents the impulse response of the pipettor as $y(0)=8, y(1)=17, y(2)=26$, etc.

According to Proposition 5 and Equation (23), the control is obtained as follows:

$$
\begin{aligned}
F_{\text {opt }} & =\left(C \bar{A}^{*} B\right) \phi\left(C \bar{A}^{*} \bar{B}\right) \phi\left(C \bar{A}^{*} \bar{B}\right) \\
& =\left[-8(9 \gamma)^{*}-4(9 \gamma)^{*}-6(9 \gamma)^{*}\right] .
\end{aligned}
$$

This feedback is not causal because there are negative coefficients in the matrix, The canonical injection from the causal elements of $\overline{\mathbb{Z}}_{\max } \llbracket \gamma \rrbracket$ (denoted $\overline{\mathbb{Z}}_{\max } \llbracket \gamma \rrbracket^{+}$) in $\overline{\mathbb{Z}}_{\text {max }} \llbracket \gamma \rrbracket$ is also residuated (see [10] for details). Its residual is given by $\operatorname{Pr}\left(\bigoplus_{k \in \mathbb{Z}} s(k) \gamma^{k}\right)=\bigoplus_{k \in \mathbb{Z}} s_{+}(k) \gamma^{k}$ where

$$
s_{+}(k)=\left\{\begin{array}{l}
s(k) \text { if }(k, s(k)) \geq(0,0) \\
\varepsilon \text { otherwise }
\end{array}\right.
$$

Therefore, the greatest causal feedback is

$$
F_{\text {opt+ }}=\operatorname{Pr}\left(F_{\text {opt }}\right)=\left[1 \gamma(9 \gamma)^{*} 5 \gamma(9 \gamma)^{*} 3 \gamma(9 \gamma)^{*}\right]
$$

The non-causal filter $P_{\text {opt }}$ is obtained by computing $\left(C \bar{A}^{*} B\right) \phi\left(C \bar{A}^{*} S\right)$ :

$$
P_{o p t}=\left(\begin{array}{cc}
-3(9 \gamma)^{*} & -8(9 \gamma)^{*} \\
1(9 \gamma)^{*} & -4(9 \gamma)^{*} \\
-1(9 \gamma)^{*} & -6(9 \gamma)^{*}
\end{array}\right)
$$

This prefilter $P_{\text {opt }}$ is not causal because there are negative coefficients in the matrix. In this example, we have Im $C \bar{A}^{*} S_{0} \subset \operatorname{Im} C \bar{A}^{*} B_{0}$, hence, this non-causal prefilter solves the MDDP and the DDP at the same time due to $C \bar{A}^{*} B_{0} P_{\text {opt }}=C \bar{A}^{*} S_{0}$. If we take the canonical injection from the causal elements of $\overline{\mathbb{Z}}_{\max } \llbracket \gamma \rrbracket$, then the greatest causal prefilter is

$$
P_{o p t+}=\operatorname{Pr}\left(P_{o p t}\right)=\left(\begin{array}{cc}
6 \gamma(9 \gamma)^{*} & 1 \gamma(9 \gamma)^{*} \\
1(9 \gamma)^{*} & 5 \gamma(9 \gamma)^{*} \\
8 \gamma(9 \gamma)^{*} & 3 \gamma(9 \gamma)^{*}
\end{array}\right)
$$

The causal filter $P_{\text {opt }}$ for the MDDP does not solve DDP because

$C \bar{A}^{*} B P_{o p t+}=\left[5(9 \gamma)^{*} 9 \gamma(9 \gamma)^{*}\right] \neq C \bar{A}^{*} S$.
Using Proposition 11, we can evaluate the difference between the solution to the MDDP and the DDP by residuation, we obtained that

$$
\begin{aligned}
\left(C \bar{A}^{*} B P_{o p t+}\right) \phi\left(C \bar{A}^{*} S\right) & =9 \gamma(9 \gamma)^{*} \\
\Delta_{Y_{1} Y_{2}} & \leq \mathcal{C}_{\left(C \bar{A}^{*} B P_{o p t+}\right) \phi\left(C \bar{A}^{*} S\right)}(0)=1 .
\end{aligned}
$$

When we apply $V=P_{\text {opt }} Q$ and $F_{o p t+}$ to the system, we can only solve for the MDDP, but not for the DDP because

$$
\begin{array}{r}
C\left(\bar{A} \oplus B F_{o p t+} C\right)^{*} B P_{o p t+}=\left[5(9 \gamma)^{*} 9 \gamma(9 \gamma)^{*}\right] \\
\neq C\left(\bar{A} \oplus B F_{o p t+} C\right)^{*} S=C \bar{A}^{*} S
\end{array}
$$

We can evaluate the distance between the two output trajectories of the output feedback controlled system and the open-loop controlled system. Using Proposition 11, we obtain that

$$
\begin{array}{r}
\left(C\left(\bar{A} \oplus B F_{o p t+} C\right)^{*} B P_{o p t+}\right) \phi\left(C\left(\bar{A} \oplus B F_{o p t+} C\right)^{*} S\right) \\
=9 \gamma(9 \gamma)^{*} \\
\Delta_{Y_{1} Y_{2}} \leq \mathcal{C}_{\left(C\left(\bar{A} \oplus B F_{o p t+} C\right)^{*} B P_{o p t+}\right) \phi\left(C\left(\bar{A} \oplus B F_{o p t+} C\right)^{*} S\right)}(0) \\
=1 .
\end{array}
$$

Notice it is the same distance compared to the openloop controlled system, because the feedback control law preserves the open-loop behaviors.

The integrated control strategy, $P_{o p t+}$ and the output feedback control $F_{\text {opt }}$, is realized in the TEG model of the HTS system, shown in Fig. 4. For example, in Fig. 4 , the first element of the causal pre-filter $P_{o p t+}(1,1)=$ $6 \gamma(9 \gamma)^{*}$ is represented by a cyclic transition due to $(9 \gamma)^{*}$, where $\gamma$ indicates one token inside of the place and 9 units are the holding time of the token, and $6 \gamma$ is represented by an additional transition with one token and 6 units holding time. Same analogy works for the feedback $F_{o p t+}: X \rightarrow U$. In order to illustrate the high throughput screening system application, an animation of the controllers for the system is online for users at the following address: http://perso-laris. univ-angers.fr/ lhommeau/automatica.html.

The Gantt chart of the scheduling is illustrated in Fig. 5. which describes the start and release time for each activity as the system evolves. For instance, without the disturbance, the system is running on its own, then it will repeat the same scheduling every 9 time units according to the TEG model, shown in the top figure in Fig. 5. For instance, the disturbance is $Q(\gamma)(1,1)=4(9 \gamma)^{*}$, which means the incubator will finish the activity 1 time unit 


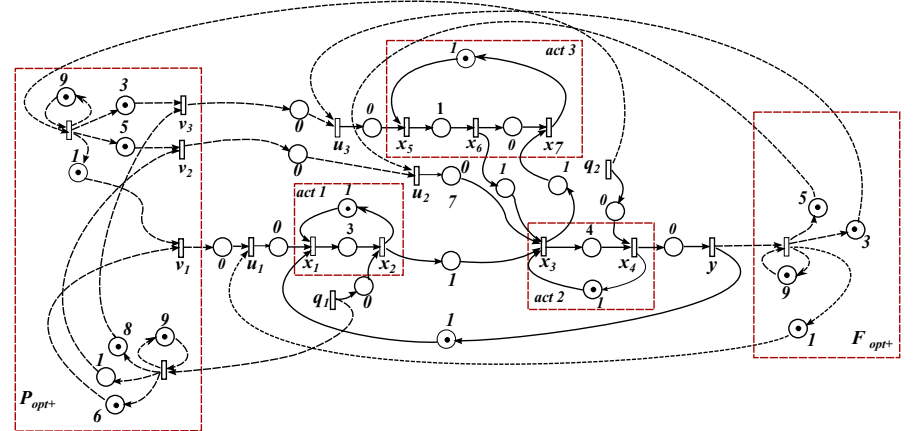

Fig. 4. The TEG model of the HTS system with the integrated controller.

late in every event cycle. In this case shown in the second figure in Fig. 5, the process time of the activity 1 at $k=0$ is 4 time unit with 1 unit delay than the original 3 time units duration, and all other events shifted 1 time unit comparing to the undisturbed system. If we implement the pre-filter $P_{o p t+}$ on the system, then the scheduling is shown in the bottom figure in Fig. 5. The MDDP is apparently solved because the start and release time of each activity with the pre-filter controller is less than or equal to the start and release time of each activity with disturbance only, shown in the middle figure. The advantage of the pre-filter is that, according to the just-in-time control principle, the activity 3 , executed on the robot, does not wait 8 time units to finish the task, and it only needs 3 time units to transport the compound B to the pipettor, without affecting the output dates. Moreover, such a pre-filter $P_{o p t+}$ proposed in this paper is designed no matter what disturbances are, which can be implemented for online processes. The integrated control strategy will preserve the same behaviors as the bottom figure in Fig. 5 .

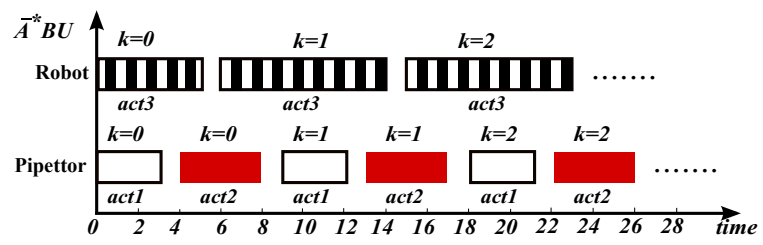

$\bar{A}^{*} S Q \uparrow$
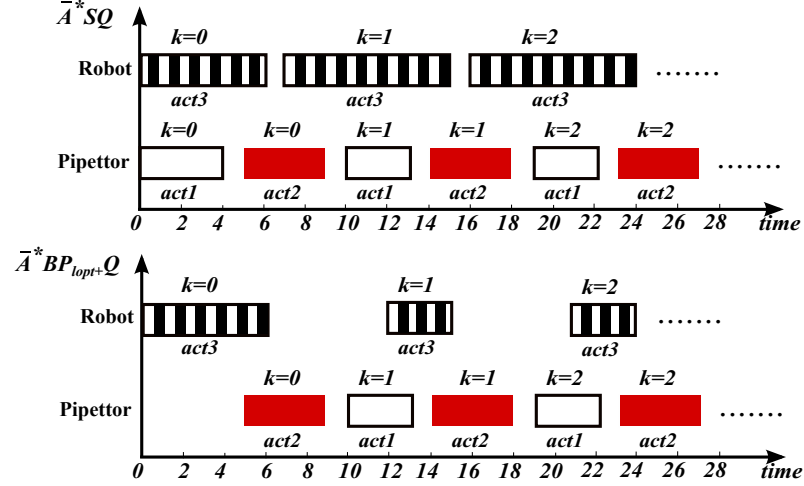

Fig. 5. The Gantt chart of the HTS system.
Moreover, when $C \bar{A}^{*} B P_{\text {opt }+} \neq C \bar{A}^{*} S$, we can find the new causal disturbance mapping $\widetilde{S}_{o p t+}$ as follows:

$$
\begin{aligned}
\widetilde{S}_{\text {opt+ }}= & \operatorname{Pr}\left(\widetilde{S}^{\text {opt }}\right)=\operatorname{Pr}\left(\left(C \bar{A}^{*}\right) \phi\left(C \bar{A}^{*} B P_{\text {opt }}\right)\right) \\
= & {\left[\begin{array}{cc}
6 \gamma(9 \gamma)^{*} & 1 \gamma(9 \gamma)^{*} \\
(9 \gamma)^{*} & 4 \gamma(9 \gamma)^{*} \\
1(9 \gamma)^{*} & 5 \gamma(9 \gamma)^{*} \\
5(9 \gamma)^{*} & 9 \gamma(9 \gamma)^{*} \\
8 \gamma(9 \gamma)^{*} & 3 \gamma(9 \gamma)^{*} \\
(9 \gamma)^{*} & 4 \gamma(9 \gamma)^{*} \\
7(9 \gamma)^{*} & 2(9 \gamma)^{*}
\end{array}\right] . }
\end{aligned}
$$

According to Proposition 13 , for any matrix $\widetilde{S} \preceq \widetilde{S}_{o p t+}$, both the DDP and the MDDP are solved by considering the same causal filter $P_{o p t+}$. For example, let $\widetilde{S}$ be a matrix with all its entries equal to $\varepsilon$ except for the non null ones of matrix $S$, i.e., the following one $\widetilde{S}(2,1)=\widetilde{S}_{\text {opt+ }}(2,1), \widetilde{S}(4,2)=\widetilde{S}_{\text {opt+ }}(4,2)$. This matrix will be such that the DDP is solvable with $P_{o p t+}$. Practically, this means that transition $x_{2}$, which was initially directly disturbed by the uncontrollable input $q_{1}$ in the original model, has to be disturbed at most by $(9 \gamma)^{*} q_{1}$, i.e. the state equation in the event domain is $x_{2}(k)=3 x_{1}(k) \oplus w_{1}(k)$ with an intermediate variable $w_{1}(k)=9 w_{1}(k-1) \oplus q_{1}(k)$. This equation can be realized as follows in the time domain $x_{2}(t)=\min \left(x_{1}(t-\right.$ $\left.3), w_{1}(t)\right)$ with $w_{1}(t)=\min \left(1+q_{1}(t-9), w_{1}(t)\right)$. The term $1+q_{1}(t-9)$ means that disturbance has to be measured and that one token has to be initially present between $q_{1}$ and $x_{2}$. If you consider $q_{1}$ as an uncontrollable component supply input, these two tokens depict the minimal stock you need to be able to solve the DDP. More generally, $\widetilde{S}_{\text {opt }}$ represents the optimal way you need to modify for the system in order to be able to reject disturbances. In the HTS system, it can represent the minimal initial stock of raw parts you need to be robust according to machine breakdown or any malfunctions in exogenous component supply.

\section{Conclusions}

This paper presents the integration of the outputfeedback controls and the open-loop controls to solve for the MDDP, as well as for the DDP. If these controls can only solve for the MDDP, not for the DDP, a criterion, based on a notion of the series distance, is established to compare the difference between two output signals solved for the MDDP and DDP, such that the performance of the controls solving for the MDDP can be evaluated. Moreover, another alternative approach is finding a new disturbance mapping in order to guarantee the solvability of the DDP by the same optimal 
control for the MDDP. Future research can be extended to other geometric control problems, such as block decoupling problem and non-interacting control problem in max-plus linear systems.

\section{Acknowledgements}

C. A. Maia is grateful for the support given by the Brazilian Agencies CNPQ, FAPEMIG and CAPES.

\section{References}

[1] F. Baccelli, G. Cohen, G.J. Olsder, and J.P. Quadrat. (1992) Synchronization and Linearity: An Algebra for Discrete Event Systems. New York: John Wiley and Sons. http://www-rocq.inria. $\mathrm{fr} / \mathrm{metalau} / \mathrm{cohen} / \mathrm{SED} / \mathrm{SED} 1-$ book.html

[2] G. Basile and G. Marro. (1969) Controlled and conditioned invariant subspaces in linear system theory, Journal of Optimization Theory and Applications, volume 3, 5, 306-315.

[3] T. Brunsch, L. Hardouin, and J. Raisch, "Control of cyclically operated high-throughput screening systems," The 10th International Workshop on Discrete Event Systems, Berlin, Germany, pp. 167172.

[4] Cohen, G.(1995) Théorie algébrique des systèmes à événements discrets, Polycopié de cours donné àl'INRIA.

[5] G. Cohen, S. Gaubert, and J.-P. Quadrat.(1996) Kernels, images, and projections in dioids, The IEEE International Workshops on Discrete Event Systems, Edinburgh, Scotland, UK. http://www-rocq.inria.fr/metalau/quadrat/ kernel.pdf

[6] G. Cohen, S. Gaubert, and J.-P. Quadrat.(1997) Linear projectors in the max-plus algebra. The 5th IEEE-Mediterranean Conference, Paphos, Cyprus. http://www-rocq.inria.fr/metalau/quadrat/ projector.pdf

[7] G. Cohen, S. Gaubert, and J.-P. Quadrat. (1999) Max-plus algebra and system theory: where we are and where to go now. Annual Reviews in Control, volume 23, 207-219.

[8] G. Cohen, S. Gaubert, and J.-P. Quadrat. (2006) Projection and Aggregation in Maxplus Algebra, Current Trends in Nonlinear Systems and Control, in Honor of Peter Kokotovic and Turi Nicosia, Boston: Birkhäuser. http: //www-rocq.inria.fr/ metalau/quadrat/Rome.pdf

[9] B. Cottenceau, L. Hardouin, M. Lhommeau, and J.-L. Boimond.(2000) Data processing tool for calculation in dioid, The IEEE International Workshop on Discrete Event Systems, Ghent, Belgium. http://www.istia.univ-angers.fr/ nardouin/outils.html
[10] B. Cottenceau, M. Lhommeau, L. Hardouin, and J.-L. Boimond.(2003) On timed event graph stabilization by output feedback in dioid, Kybernetika, volume 39, 2, 165-176.

[11] J.S. Golan.(1999) Semirings and Their Applications. Boston: Kluwer Academic Publishers..

[12] L. Hardouin, C.A. Maia, B. Cottenceau, and M. Lhommeau.(2010) Observer Design for (max,plus)-Linear Systems, IEEE Transactions on Automatic Control, volume 55, 2, 538-543.

[13] L. Hardouin, M. Lhommeau, and Y. Shang.(2011) Towards Geometric Control of Max-Plus Linear Systems with Applications to Manufacturing Systems, The 50th IEEE Conference on Decision and Control and European Control Conference, 11491154, Orlando, FL. USA.

[14] R.D. Katz.(2007) Max-plus (A,B)-invariant spaces and control of timed discrete-event systems, IEEE Transactions on Automatic Control, volume 52, 2, 229-241.

[15] J.-Y. Le Boudec, and P. Thiran.(2002) Network Calculus. New York: Springer-Verlag.

[16] M. Lhommeau, L. Hardouin and B. Cottenceau.(2002) Disturbance decoupling of timed event graphs by output feedback controller, The 6th International Workshop on Discrete Event Systems, Zaragoza, Spain. http://www.istia. univ-angers.fr/ hardouin/Wodes02.pdf

[17] M. Lhommeau, L. Hardouin, and B. Cottenceau. (2002) About disturbance decoupling of timed event graphs in dioids. The 6th International Workshop on Discrete Event Systems, Zaragoza, Spain, 203-208. http://www.istia. univ-angers.fr/ hardouin/Wodes02.pdf

[18] M. Lhommeau, L. Hardouin and B. Cottenceau.(2003) Optimal control for $(\max ,+)$-linear systems in the presence of disturbances, Positive Systems: Theory and Applications, POSTA, Springer LNCIS 294,47-54.

[19] M. Di Loreto, S. Gaubert, R. Katz, and JJ. Loiseau. (2010) Duality between invariant spaces for max-plus linear discrete event systems, SIAM Journal on Control and Optimization, volume. 48, 5606-5628. http://fr.arXiv.org/abs/ 0901.2915

[20] C.A. Maia, L. Hardouin, R. Santos-Mendes, and B. Cottenceau.(2005) On the Model reference control for max-plus linear systems. The 44th IEEE Conference on Decision and Control, 7799-7803.

[21] C.A. Maia, C.R. Andrade, and L. Hardouin.(2011) On the control of max-plus linear system subject to state restriction. Automatica, volume 47, 5, 988992.

[22] MaxPlus Group.(1991) Second order theory of minlinear systems and its application to discrete event systems. Proceedings of the 30th IEEE Conference on Decision and Control.

[23] J.-M. Prou and E. Wagneur.(1999) Controllability in the max-algebra. Kybernetika, volume 35, 1, 13- 
24.

[24] R. Santos-Mendes, B. Cottenceau, and L. Hardouin.(2005) Adaptive feedback control for (max,+)-linear systems. The 10th IEEE International Conference on Emerging Technologies and Factory Automation, volume. 2, 164-170. http: //dx.doi.org/10.1109/ETFA.2005.1612675

[25] Y. Shang, L. Hardouin, M. Lhommeau and C.A. Maia.(2013) Open-loop controllers to solve the disturbance decoupling problem for max-plus linear systems, 2013 European Control Conference, Zurich, Switzerland, 2013.

[26] W.M. Wonham.(1979) Linear Multivariable Control: A Geometric Approach. New York: SpringerVerlag.

\section{A Formulas of Star Operations}

\begin{tabular}{cc}
\hline$a^{*}\left(b a^{*}\right)^{*}=(a \oplus b)^{*}=\left(a^{*} b\right)^{*} a^{*}$ & (f.1) \\
$\left(a^{*}\right)^{*}=a^{*}$ & (f. 2$)$ \\
$(a b)^{*} a=a(b a)^{*}$ & (f.3) \\
$a^{*} a^{*}=a^{*}$ & (f. 4$)$ \\
$a a^{*}=a^{*} a$ & (f.5) \\
\hline
\end{tabular}

B Formulas of Left Residuations

\begin{tabular}{cc}
\hline$a(a \phi x) \preceq x$ & (f.6) \\
$a \phi(a x) \succeq x$ & (f.7) \\
$a(a \phi(a x))=a x$ & (f.8) \\
$a \phi(x \wedge y)=a \phi x \wedge a \phi y$ & (f.9) \\
$(a \oplus b) \phi x=a \phi x \wedge b \phi x$ & (f.10) \\
$(a b) \phi x=b \phi(a \phi x)$ & (f.11) \\
$b(a \phi x) \preceq(a \phi b) \phi x$ & (f.12) \\
$(a \phi x) b \preceq a \phi(x b)$ & (f.13) \\
\hline
\end{tabular}

C Formulas of Right Residuations

\begin{tabular}{cc}
\hline$(x \phi a) a \preceq x$ & (f.14) \\
$(x a) \phi a \succeq x$ & $(\mathrm{f} .15)$ \\
$((x a) \phi a) a=x a$ & $(\mathrm{f} .16)$ \\
$(x \wedge y) \phi a=x \phi a \wedge y \phi a$ & (f.17) \\
$x \phi(a \oplus b)=x \phi a \wedge x \phi b$ & $(\mathrm{f} .18)$ \\
$x \phi(b a)=(x \phi a) \phi b$ & (f.19) \\
$(x \phi a) b \preceq x \phi(b \phi a)$ & (f.20) \\
$b(x \phi a) \preceq(b x) \phi a$ & (f.21) \\
\hline
\end{tabular}

